\title{
IMAGE SEGMENTATION OF MRI IMAGE FOR BRAIN TUMOR DETECTION
}

\author{
Asim Zaman, Kifayat Ullah, Raza Ullah, Hafiz Hasnain Imtiaz \\ Postgraduate Students in School of Electronics and Information, \\ Liaoning University of technology, Jinzhou, P.R China \\ Dr. Ling Yu \\ Associate Professor in School of Electronics and Information, \\ Liaoning University of technology, Jinzhou, P.R China
}

\begin{abstract}
- this research work presents a new technique for brain tumor detection by the combination of Watershed algorithm with Fuzzy K-means and Fuzzy C-means (KIFCM) clustering. The MATLAB based proposed simulation model is used to improve the computational simplicity, noise sensitivities, and accuracy rate of segmentation, detection and extraction from MR images of brain tumor. The preprocessing stage consists of denoising, skull stripping and image enhancement, after which MR images are segmented specially by using watershed algorithm followed by Fuzzy K-means and Fuzzy C-means (KIFCM) clustering algorithm. The experimental results of the proposed idea are also compared to the fuzzy C-mean, K-means, Maximization Expectation, and Mean Shift. Superiority of the proposed technique is evaluated through qualitative and quantitative validation experiments in term of noise sensitivity, capture range, computational simplicity and segmentation accuracy.
\end{abstract}

Keywords - Medical image segmentation, MRI, Brain tumor, Watershed segmentation, K-means clustering, Fuzzy C-means and K-mean

\section{INTRODUCTION}

The diagnosis of brain tumor cell is very complicated due to sensitive organ of human body [1]. The early diagnosis and detection of tumor cell is very important [2]. However, the detection process of tumor area is very complicated and challenging task due to the variance in size, shape, location and intensity levels of tumor images [3][4].

Image segmentation is the process of partitioning digital images into multiple regions and parts to analyze and find the location and boundaries of images more easily [5]. Image segmentation is also called the partitioned study of entire image. Therefore, the image segmentation plays very important role in medical field of diagnosis of MR images. Due to poor contrasts, noise sensitivities and diffusive boundaries in MR images, segmentation is important [6].
Magnetic Resonance Imaging (MRI) and computed tomography (CT) are the two scanning methods of tumor cells. The Magnetic Resonance Imaging (MRI) considered more confortable due to lack of radiation and high accuracy rate [7]. That's why it's very important to choose the more proper way of diagnosis.

Image segmentation techniques are based on two essential image intensity factors (irregularity and similarities) [8]. The segmentation technique in the formal classification is based on splitting the generated image on the basis of intensity changes such as edges and corners. The second is based on dividing an image into relative region due to set of pre-defined parameters. There are several segmentation techniques and methods that can be used widely, such as histogram based approach, artificial neural network, edge-based methods, region-based (growing, splitting and merging), physical model-based approaches, and clustering-based techniques( $\mathrm{k}$ means, Fuzzy C-means, Expectation Maximization and Mean shift) [8]-[10].

Image segmentation has many challenging issues, such as creating a cohesive approach which can be implemented to all images and application types, while finding a suitable technique for a particular type of image is a challenging issue. Therefore, for image segmentation, there is no universally accepted method in the fields of image processing and computer vision [11].

One perspective of image segmentation is a clustering issue that involves the most appropriate way of determining which pixels belong together in an image. Tools that conduct image segmentation based on clustering methods are listed extensively. Generally, such methods represent clustering by either partitioning or grouping pixels in one of the two various ways. The whole object is split into regions that are "successful" according to certain requirements, while partitioning of the images. Hence, the pixels are placed together in the grouping based on certain assumptions [12]. There are several clustering techniques which can be used in the processes of image segmentation, like hard or fast clustering, clustering with K-means, and clustering with Fuzzy 


\section{International Journal of Engineering Applied Sciences and Technology, 2019 Vol. 4, Issue 8, ISSN No. 2455-2143, Pages 50-55 \\ Published Online December 2019 in IJEAST (http://www.ijeast.com)}

C-means. Clustering is also a complicated field. It's being used as a stand-alone method for further research to gain knowledge and to insight, into the processing of the information in various clusters [13].

To identify the brain tumor and measure the tumor region, we used image segmentation techniques based on the watershed algorithm along with clustering approach. For effected MRI images, we introduced a new model segmentation method called K-means merged with Fuzzy C-means (KIFCM).

We implemented K-means with the Fuzzy C-means algorithm after the initial stage of the pre-processing and watershed algorithm to address the deficiencies and benefits of it. After clustering, the tumor is automatically removed without user interaction. And to utilize thresholding and level-set methods for crease the brain tumor region. Calculating the tumor region in the analyzed image is the last stage of the developed methodology. K-means algorithm can identify a tumor in the brain more easily than Fuzzy C-means. Nevertheless, tumor cells that are not defined by K-means algorithm are determined by Fuzzy C-means. Compared to the K-means algorithm and $\mathrm{C}$-means, the proposed approach gives an accurate performance.

\section{IMAGE SEGMENTATION: RELATED WORK}

Image segmentation is a challenging task in medical image processing. Many techniques and algorithms have invented by researchers. For example, Watershed and Thresholding segmentation are more efficient and reliable to detect abnormal part in medical images. The work done by Patil and Bhalchandra [14], the images were firstly read by the algorithms and then converted into gray images for further processing. To find the tumor images boundaries and denoising, high pass filter was applied. Furthermore, the morphological operations were used to find the size and location of tumor. The watershed segmentation along with morphological operation is also one of the simplest ways to detect tumor from MRI images. The preprocessing stage consists of skull stripping, de-noising and image enhancement. The proposed method was based on marked controlled watershed segmentation, and morphological operations were used for exact location of tumor. Furthermore, frustum model was used for tumor area calculation [15]. Hoseynia et al [16] proposed a model by using watershed along with fuzzy cmean for edge detection of brain tumor in MR images. The results and accuracy rate were quite impressive as compare to individual used of watershed segmentation and fuzzy C-mean. They used fuzzy c-mean algorithm after preprocessing stage and followed by watershed markers. Bandhyopadhyay and Paul [17] have proposed a K-mean based clustering algorithm for the brain tumor segmentation of MR images. The proposed method has identified and classified the brain tumor by using K-mean segmentation algorithm, coarse gain and fine grain localization. The classification of data set based on two segments of array, one segment contains the set of normal brain cells (Grey Matter, White Matter and Cerebral Spinal Fluid), while the other set consists of tumor cells. Glavan and Holban [18] have proposed a convolution neural network (CNN) based pixel classifier for the segmentation analysis of X-ray images. The system was designed to classify them into bone and non-bone tissues. The separation of bone tissues area from the original image was the main point of the proposed work. Compare to the other configurations their method provided a good results for limited number of data, but the main problem of the proposed research was, it takes higher execution time and provided irregularities in the bone area.

Dubey and Yerpude [19] have developed an efficient KMedoids Clustering segmentation technique for brain tumor detection. The proposed model worked based on Medoid. KMedoids were used as a reference point rather than mean value to find the cluster point of the objects. The centroids were found accurately but the only problem was found in optimal number of segments. The K-means Clustering algorithm provided better results as compare to Hierarchical clustering and K-Mediods [20].In this paper, we applied watershed segmentation technique along with KIFCM. We also analyzed and compared the accuracy based performance of the fuzzy C-means, K-means, Maximization Expectation, Mean Shift and KIFCM. The main idea is based on accuracy, execution time, over-segmentation, under-segmentation and computational simplicity.

\section{THE PROPOSED APPROACH}

Several clinical image segmentation systems are available that have used the K-means algorithm to detect tumor tissue in the brain. The K-means algorithm allows easy and faster way to execute onto large databases, but somehow it declines with inaccurate tumor identification, specifically when it is a malignant tumor tissue. While, Fuzzy C-means algorithm provides the detail information's of the image to recognize malignant tumor reliably relative to a K-means. The proposed image segmentation approach consists of Pre-Processing (Denoising, Skull-stripping and Image enhancement), PostProcessing (watershed and KIFCM), extraction and validation phases as shown in Fig. 1. Our experimental results have confirmed that watershed algorithm along with (KIFCM) could identify and detect the brain tumor more accurately. 


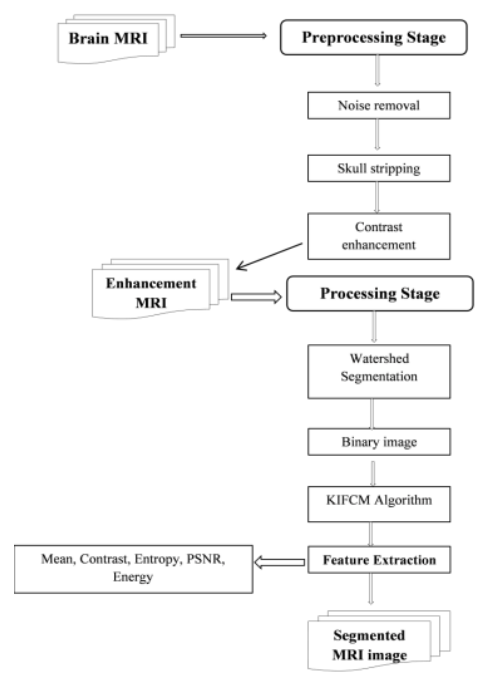

Fig. 1. The framework of the proposed image segmentation system

\section{A. Preprocessing -}

De-noising: it has been used to eliminate the internal noise, and to enhance the fine details of the input image. Several filters are used to reduce the noise from images, such as, Gaussian, linear and average filter [21]. We used median filter in our work because of high noise sensitivities.

Skull removal: Normally the background image does not contain certain valuable information. However, the processing time is increased. Eliminating background, eyes, scalp and all non-interesting structures will reduce the amount of memory used and maximize processing speed. BSE (brain surface extractor) algorithm is used to remove the skull from the input MR images. BSE algorithm is used only for MRI [22]. To extract abnormalities, it filters the image, identifies boundaries throughout the image and implements the morphological erosions.

MRI enhancement: It can be accomplished for contrast enhancement by using various operators of computational morphology or wavelet transformation [23]. We used Gaussian high-pass filter here to boost the boundaries of the image structures.

\section{B. Segmentation Phase -}

We proceed to segmentation with two approaches after enhancing images: the watershed and the KIFCM method.

\section{Watershed Segmentation -}

The Watershed transformation can be classified as a region based segmentation approach. The idea intuitive behind this method comes from geography: it is that of a landscape or topographic relief which is flooded by water. Watersheds being break lines of attraction areas of rain falling on the area. As a result, the region is partitioned into basins separated by dams, called watershed lines or simply watersheds. More details are found in [24]. We used this watershed segmentation approach before clustering stage in our work.

The transformation of the Watershed can be defined as an approach to segmentation based on area and region. The conceptual idea behind this approach comes via geography: it is the concept of a water-flooded landscape or topographical escape. Watersheds are breaking routes of rain dropping arousal regions on the target area. As a result, the zone is separated into watershed segments or literally watersheds, separated by dams. More details are found in [25]. We used this watershed segmentation approach before clustering stage in our work.

\section{Clustering Phase -}

The images are fed to the KIFCM technique after the segmentation stage of Watershed by initializing cluster numbers $\mathrm{k}$, max iterations. The cluster centers are calculated by:

$$
M \mu=((1: K) * m) /((k+1))
$$

$M \mu$, Is the initial mean, $\mathrm{K}$ is the cluster number and $\mathrm{m}$ is defined as:

$$
M \mu=\max (\text { MRI image })+1
$$

Therefore, by testing the distance between the point and the cluster centers, assign the position to the closest cluster center based on a shortest distance; instead re-compute the new cluster centers [26]-[28]. On the other hand, some areas are distributed for away from each cluster center. The corresponding existing cluster points, clustered positions and distributed positions could be accessed simultaneously with the loop step that evaluating the new intervals and clustering the points [27]. The participation attributes and means are then modified to determine the closing condition.

The looping route takes less iteration duration than random sampling even though the cluster's initial centers were not selected randomly, minimizing effort and time. However, due to membership, the points are re-clustered. There is no difference in their clusters between points because the reclustering process does not make a huge change. The technique output is the clustering image, time of execution, and numbers of iteration that are reported to compare with other methods of clustering. We are making a hybrid clustering approach at this point focused on soft and hard clustering. The technique of hard clustering allows each point to contribute only to the nearest cluster. The soft clustering method, on the other hand, offers each point a standard of participation instead of contributing entirely to one group.

\section{Extraction Stage-}

One of the essential techniques of image processing and computer vision is thresholding or object binarization. To extract the object from the background binarization and 


\section{International Journal of Engineering Applied Sciences and Technology, 2019 \\ Vol. 4, Issue 8, ISSN No. 2455-2143, Pages 50-55 \\ Published Online December 2019 in IJEAST (http://www.ijeast.com)}

thresholding are used. The segmented image obtained through thresholding does have the attributes of less storage capacity, rapid reaction time and simplicity of distortion relative to the gray-level image which typically includes a huge amount of gray levels [29]. The output segmented images contain tumor area (lighting area) and non-tumor area (dark background). The area of the tumor is determined by measuring the white pixels of the image.

\section{EXPERIMENTAL RESULTS AND DISCUSSION}

We used two tumor and non-tumor data sets to test the efficiency of the image segmentation approach by Using MATLAB (R2017a); Figure 2 illustrates the various results got during the process of segmentation. The first line (a) illustrates our system's original brain MR images, each containing a tumor. (b) De-noising and skull stripping. (C) Represents the tumor image gradient. (D) The watershed strategy works. (E) KIFCM (Fuzzy K-means and Fuzzy Cmeans) final segmented images (f). The tumor region corresponds to the white area. We use two quantitative metrics for assessing the efficiency of the segmentation, Peak Signal-to-Noise Ratio (PSNR) and Structural Similarity (SSIM).

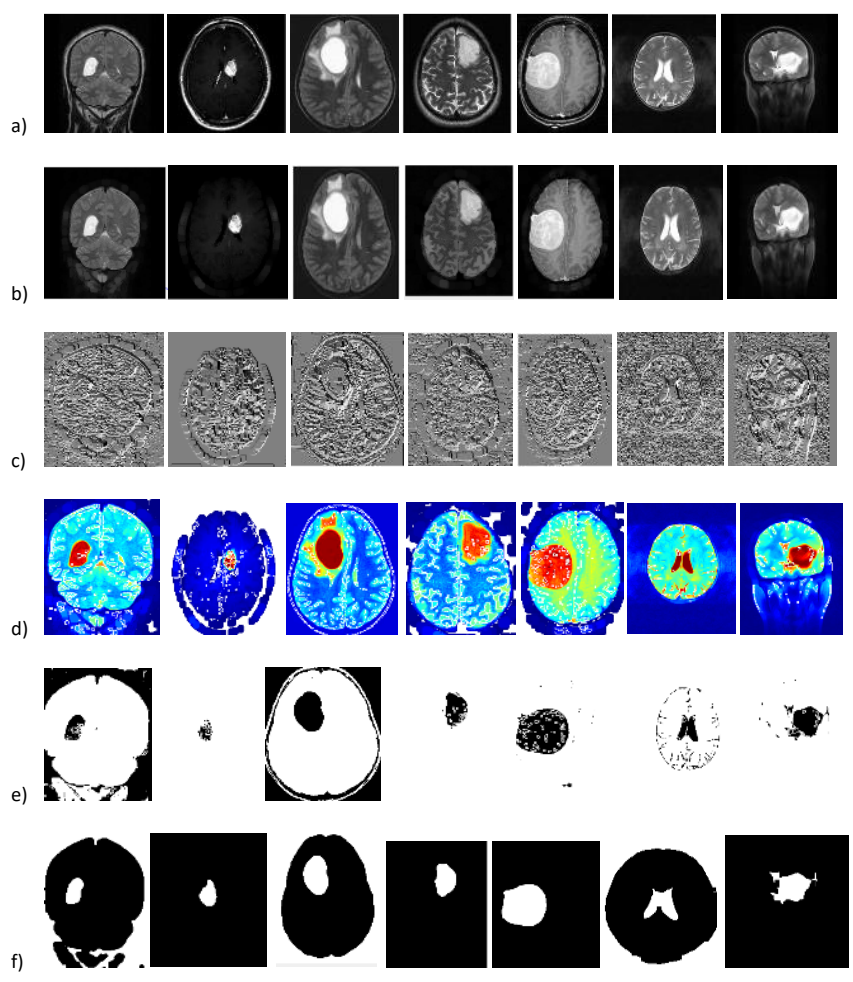

Fig. 2. Results of our proposed approach: (a) original brain MR images (b) de-noising and skull stripping (c) Gradient MR images (d) watershed segmentation (e) KIFCM (f) tumor image
We find in some images that the KIFCM approach is more reliable than Fuzzy C-mean, as shown in Table 01. Due to high number of iteration in FCM which cause overlapping in resulting image, however, by using the KIFCM for the same data set, the number of iteration is reduced with successful results. The comparison was made between the five approaches evaluated on the basis of the specific performance metrics as shown in table 01 .

$$
\begin{aligned}
& \text { True positive }(\mathrm{TP})=\frac{\text { No of resulted tumor images }}{\text { totlal No of images }} \\
& \text { True Negative }(\mathrm{TN})=\frac{\text { No of non-tumor images }}{\text { totlal No of images }} \\
& \text { False positive }(\mathrm{FP})=\frac{\text { No of non-tumor images but detected positive }}{\text { totlal No of images }} \\
& \text { False Negative }(\mathrm{FN})=\frac{\text { No of tumor images but detected negitive }}{\text { totlal No of images }} \\
& \text { Precision }=\left[\frac{\mathrm{True} \text { positive }(\mathrm{TP})}{(\mathrm{True} \text { positive }(\mathrm{TP})+\mathrm{False} \text { positive }(\mathrm{FP}))}\right] \\
& \text { Recall }=\left[\frac{\text { True positive }(\mathrm{TP})}{(\text { True positive }(\mathrm{TP})+\mathrm{False} \text { negative }(\mathrm{FN}))}\right] \\
& \text { Accuracy }=\left[\frac{\text { True positive }(\mathrm{TP})+\text { True Negative }(\mathrm{TN})}{((\mathrm{TP})+(\mathrm{TN})+(\mathrm{FP})+(\mathrm{FN}))}\right]
\end{aligned}
$$

\begin{tabular}{|c|c|c|c|c|c|c|c|}
\hline \multicolumn{8}{|c|}{ Clustering Techniques } \\
\hline \multicolumn{8}{|c|}{ KIFCM Fuzzy K-means and Fuzzy C-means (KIFCM) } \\
\hline Data sets & TP & TN & FP & $\mathrm{FN}$ & Accuracy & Precision & Recall \\
\hline D1 & 91 & 0 & 0 & 7.5 & 92.10 & 100 & 92.10 \\
\hline \multicolumn{8}{|c|}{ Fuzzy C Mean (FCM) } \\
\hline Data sets & TP & TN & FP & $\mathrm{FN}$ & Accuracy & Precision & Recall \\
\hline D1 & 86.6 & 0 & 0 & 13 & 86.6 & 100 & 86.6 \\
\hline \multicolumn{8}{|c|}{$\mathrm{K}$-means $(\mathrm{KM})$} \\
\hline Data sets & TP & TN & FP & $\mathrm{FN}$ & Accuracy & Precision & Recall \\
\hline D1 & 86 & 0 & 0 & 14.6 & 85.48 & 100 & 85.48 \\
\hline \multicolumn{8}{|c|}{ Mean shift (MS) } \\
\hline Data sets & TP & $\mathrm{TN}$ & FP & $\mathrm{FN}$ & Accuracy & Precision & Recall \\
\hline D1 & 88 & 0 & 0 & 14.2 & 86.1 & 100 & 86.1 \\
\hline \multicolumn{8}{|c|}{ Expectation Maximization (EM) } \\
\hline Data sets & TP & $\mathrm{TN}$ & FP & $\mathrm{FN}$ & Accuracy & Precision & Recall \\
\hline D1 & 69 & 0 & 0 & 34 & 66.9 & & 66.7 \\
\hline
\end{tabular}

Table -1 The Comparative results of clustering techniques 


\section{International Journal of Engineering Applied Sciences and Technology, 2019 Vol. 4, Issue 8, ISSN No. 2455-2143, Pages 50-55 \\ Published Online December 2019 in IJEAST (http://www.ijeast.com)}

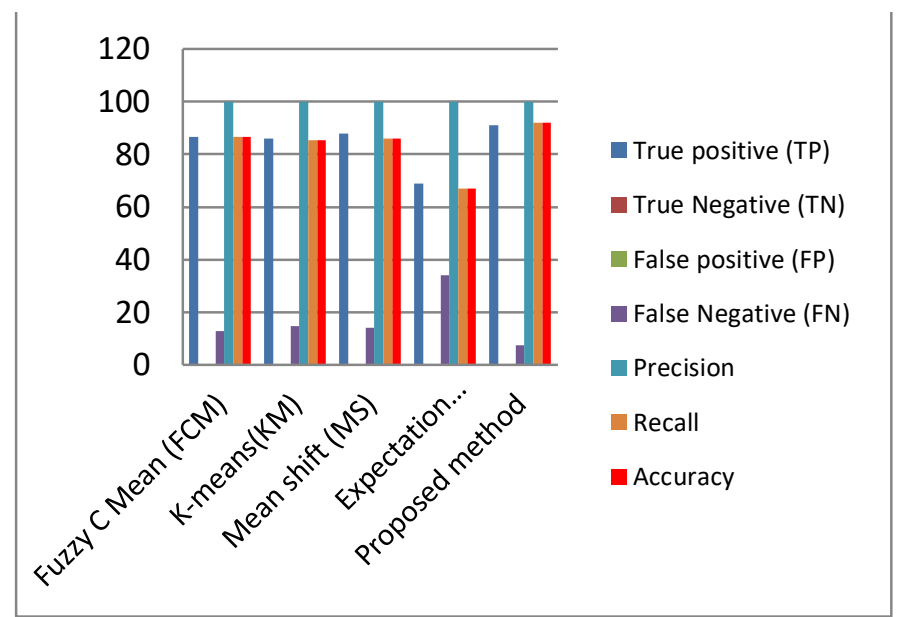

Fig. 3. The comparative result of clustering techniques based on accuracy

From the above table 01 and figure 03, our suggested technique is obviously the most effective with minimum execution time.

\section{CONCLUSION}

We also noted that the watershed is better than quantitative morphology. We have developed a new method, Watershed segmentation along with (KIFCM), which combines the Fuzzy $\mathrm{C}$-means clustering algorithm with the K-means to diagnose brain tumors effectively in minimum execution time. Our approach consists of the following stages: pre-processing (denoising and skull removal), watershed algorithm, clustering technique (K-means and Fuzzy C-means integration) and extraction. The experimental results of the proposed idea are also compared to the fuzzy C-mean, K-means, Maximization Expectation, and Mean Shift. Superiority of the proposed technique is evaluated through qualitative and quantitative validation experiments in term of noise sensitivity, capture range, computational simplicity and segmentation accuracy. In order to reduce the execution time, our proposed solution specifies the initial cluster $\mathrm{k}$ value. The 3D assessment of brain tumor identification using 3D slicer would be done in future work.

\section{REFERENCE}

[1] A. Dhanuskodi and K. Perumal, "ovel Approach for Noise Removal of Brain Tumor MRI Images," Journal of Biomedical Engineering and Medical Imaging, vol. 2, no. 3, 2015.

[2] K. Somasundaram and K. Ezhilarasan, "Automatic Brain Portion Segmentation From Magnetic Resonance Images of Head Scans Using Gray Scale Transformation and Morphological Operations," ournal of Computer Assisted Tomography, vol. 39, no. 4, pp. 552-558, 2015.
[3] S. .zu, "brain tumor detection and segmentation using watershed segmentation and morphological operation"," International Journal of Research in Engineering and Technology, vol. 3, no. 3, pp. 367-374, 2014.

[4] B. Sayah and B. Tighiouart, "Brain tumour segmentation in MRI: knowledge-based system and region growing approach," International Journal of Biomedical Engineering and Technology, vol. 14, no. 1, p. 71, 2014.

[5] "Brain Tumor Detection and Segmentation by Fuzzy CMeans (FCM) Algorithm Using LabVIEW," International Journal of Recent Trends in Engineering and Research, vol. 4, no. 2, pp. 104- 107, 2018.

[6] A. Chien, B. Dong and Z. Shen, "Frame-based segmentation for medical images," Communications in Mathematical Sciences, vol. 9, no. 2, pp. 551-559, 2011.

[7] A. Chien, B. Dong and Z. Shen, "Frame-based segmentation for medical images," Communications in Mathematical Sciences, vol. 9, no. 1, pp. 1-5, 2019.

[8] G. K and D. P, "Review on MRI Brain Tumor Segmentation Approaches," Bonfring International Journal of Advances in Image Processing, 2019.

[9] R. Oak, "A Study of Digital Image Segmentation Techniques," International Journal Of Engineering And Computer Science, 2016.

[10] P. SrinivasaRao, K. Suresh and B. R. Kiran, "mage Segmentation using Clustering Algorithms," International Journal of Computer Applications, vol. 120, no. 14, pp. 36-38, 2015.

[11] A. C. S, m. K and k. A, "improved hybrid segmentation of brain mri tissue and tumor using statistical features," ctact journal on image and video processing, vol. 1, no. 1, pp. 43-49, 2010.

[12] G. panchal, "Analysis of Image Segmentation Techniques," International Journal of Recent Advancement in Engineering \& Research, vol. 3, no. 3, p. 4, 2017.

[13] W. Khan, "mage Segmentation Techniques: A Survey," Journal of Image and Graphics, pp. 166-170, 2014.

[14] M. Panda, "some clustering algorithms to enhance the performance of the network intrusion detection system," journal of Theoretical and Applied Information Technology, vol. 26, no. 2, pp. 710-716, 2008.

[15] O. S. Zin and A. S. Khaing, "Brain Tumor Detection and Segmentation Using Watershed Segmentation and Morphological Operation," IJRET Int. J. Res. Eng. Technol, vol. 3, no. 3, pp. 367-374, 2014.

[16] M. Sharma and S. Mukherje, "Fuzzy cmeans, anfis and genetic algorithm for segmenting astrocytoma-a type of brain tumor," IAES International Journal of Artificial Intelligence, vol. 3, no. 1, p. 16, 2014.

[17] M. A and R. K, "Spatial Fuzzy C-means PET image segmentation of neurodegenerative disorder spatial Fuzzy 


\section{International Journal of Engineering Applied Sciences and Technology, 2019 \\ Vol. 4, Issue 8, ISSN No. 2455-2143, Pages 50-55 \\ Published Online December 2019 in IJEAST (http://www.ijeast.com)}

C-means PET image segmentation of neurodegenerative disorder," Indian J Comput Sci Eng (IJCSE), vol. 4, no. 1, p. 50, 2013.

[18] T. S and M. A, "Image Segmentation using k means clustering, EM and normalized Cuts," University Of California Irvine, technical report.

[19] Y. A and D. S, " Colour image segmentation using Kmedoids clustering," Int J Comput Technol Appl, vol. 3, no. 1, p. 152, 2012.

[20] W. B and D. JPM, "An experimental analysis of Fuzzy Cmeans and K-means segmentation algorithm for iron detection in brain SWI using Matlab," Int J Comput Appl, vol. 104, no. 15, pp. 36-8, 2014.

[21] K. L and T. S, "A survey on brain tumor detection using image processing technique," International Conference on Cloud Computing. IEEE, 2007.

[22] H. W. L, Y. M. S and C. D. H, "Parameter selection for suppressed fuzzy c -means with an application to MRI segmentation," Pattern Recognition Letters, vol. 27, no. 5, pp. 424-438, 2006.

[23] A. S. U, G. G and D. D, "A Survey on Detection of Brain Tumor from MRI Brain Images," International Conference on Control, Instrumentation, Communication and Computational Technologies. IEEE, 2014.

[24] K. L and T. S, "A survey on brain tumor detection using image processing techniques," International Conference on Cloud Computing. IEEE, 2017.

[25] W. Z, X. W and Z. L. I, "Brain tumor image segmentation based on C- V model optimized by watershed transformation," Computer Engineering \& Applications, 2017.

[26] P. G. B and A. A, "Hybrid approach for brain tumor detection and classification in magnetic resonance images[," Communication, Control \& Intelligent Systems. IEEE, 2016.

[27] S. M, Y. G and L. T, "Supervised Learning based Multimodal MRI Brain Tumour Segmentation using Texture Features from Supervoxels," Computer Methods \& Programs in Biomedicine, 2018.

[28] s. R and D. M, "Image segmentation for uneven lighting images using adaptive thresholding and dynamic window based on incremental window growing approach," Int J Comput App, vol. 56, no. 13, pp. 31-36, 2012.

[29] L. CP, "obust image segmentation using active contours," level set approache, PhD thesis, Department of Electrical and Computer Engineering North Carolina State University, 2005. 